\title{
HERB-BASED FUNCTIONAL FOODS: FROM LABORATORIES TO THE MARKET
}

\section{(C) Tsay Hsin-Sheng ${ }^{1,2}$}

'Institute of Biochemical Science and Technology, Chaoyang University of Technology, Wufong, Taichung, Taiwan ${ }^{2}$ Department of Agronomy, College of Agriculture and Natural Resources, National Chung Hsing University, Taichung, Taiwan

Consumption of alternative herbal folk medicine has a tremendous increase in the last decade. A number of medicinal plants contain secondary metabolites which have many biologically active compounds. They are used against hepatic fibrosis and heart ischemia-reperfusion and proved to have antioxidant, antithrombosis, antihypertension, antistress, antivirus, antitumour, antiulcer, antidiabetic, antiaging, and anti-inflammatory activities. Non-availability of quality planting materials, low germination, and slow plant growth, disease and pest incidence are the major obstacles in conventional medicinal plant cultivation. In Taiwan, many economically important medicinal plants and herbs are produced using various explant materials by tissue culture technique to meet the increasing demand for their medicinal properties. Rapid multiplication through in vitro tissue culture can be advantageous for the continuous supply throughout the year. We have developed and standardized efficient, simple and rapid tissue culture regeneration protocols of many medicinal plants, optimized the conditions in green house and successfully established the regenerated plantlets in the field for the large scale commercial production. Availability of tissue culture protocol is the first step towards the development of the genetic transformation.

Acknowledgement: Thanks to the National Science Council of Taiwan for financial support.

\section{CHEMICAL CONSTITUENTS FROM HERBA CIATANCHE AND THEIR EFFECTS ON NEURODEGENERATIVE DISEASE}

\section{(C) Tu Pengfei}

State Key Laboratory of Natural and Biomimetic Drugs, School of Pharmaceutical Sciences, Peking University Health Science Center, Beijing 100191, China

Herba Cistanche (Rou Cong Rong), dried succulent stems of Cistanche species, is a famous Chinese herbal medicine used for the treatment of kidney deficiency, female infertility, morbid leucorrhea, neurataxia and senile constipation, etc. There are four species and one variety of Cistanche in China, i.e.C. deserticola, C. tubulosa, C. salsa, C. salsa var. albiflora and $C$. sinensis. A systematic chemical investigation has been performed on these Cistanche species, and 118 compounds were isolated and identified. Among them, 34 are new compounds, including 17 new phenylethanoids and 17 new polysaccharides. The total phenylethanoid glycosides from $C$. tubulosa showed good protective and therapeutic effects for senile dementia in several animal models, for example, it improved the learning and memory in mice, protected the memory recurrence dysfunction of mice induced by ethanol, and the memory acquirement dysfunction of mice induced by Scopolamine, and showed a good melioration effect on learning and memory ability dysfunction of focal cerebral ischemia-reperfusion dementia rat. Based on these, the total phenylethanoid glycosides from $C$. tubulosa have been developed to a new drug called "Congrong Zonggan Capsule" for curing vascular dementia and Alzheimer's disease. Up to now, three clinical trials have been finished, and the syndrome improvements were 84.4 and 91.2 in II and III clinical trials, respectively. The mechanism study showed that phenylethanoids, such as echinacoside and acteoside could reduce intracellular reactive oxygen species levels, inhibit the activity of caspase-3 protease in apoptosis pathway and maintain the mitochondrial membrane potential in high-energy state to play the neuroprotective role. 